\title{
Towards robust service workflows: a decentralized approach
}

\author{
Mario Henrique Cruz Torres and Tom Holvoet \\ Department of Computer Science \\ Katholieke Universiteit Leuven \\ Leuven, Belgium \\ \{MarioHenrique.CruzTorres, Tom.Holvoet\}@cs.kuleuven.be
}

\begin{abstract}
Nowadays service workflows are used to increase the efficiency of process oriented supply chains. Service workflows can encompass hundreds of services around a single process. These services are geographically spread and cross organizational boundaries. This raises the need for coordination, such as assigning tasks, synchronizing production schedules, between companies collaborating through services. We present a fully decentralized coordination mechanism that, using the local knowledge available at each company participating in the supply chain, allows the enactment of robust processes. We evaluate our solution through simulations and show that it can create robust service compositions.
\end{abstract}

\section{Introduction}

Nowadays the business world faces an increasing pressure to quickly create new products and offer them at lower prices. In order to achieve these goals companies focus on their core specialties and at the same time collaborate with a wide range of suppliers. Several companies organize themselves around processes, creating process oriented supply chains. On the one hand process oriented supply chains help companies to improve their efficiency, be more agile and responsive to market changes. On the other hand, process oriented supply chains demand better communication, even more, better collaboration amongst its participants [1]. Cooperation is a cornerstone to enable process oriented supply [2].

Companies can expose their services on the internet. However there is no simple way to define how multiple services can interact with each other in order to improve the overall efficiency of a system, or of a process. There are studies proposing how services can share resource information in standardized ways [3]. However there is a lack of mechanisms to specify how services interact, which information to share, and how to monitor cross-organizational business processes.

Our main contribution is to present a decentralized coordination mechanism that selects services to participate in a supply chain process. Our mechanism, based on Delegate MAS [4], is a step in the direction of decentralized services in supply chains.

The rest of the paper is organized as follows. Section 2 describes the problem explored. We present our solution in Section 3 and evaluate it on Section 4. Finally, Section 5 shows the related works and Section 6 our conclusions. 


\section{Service Selection in Supply Chains}

The Supply Chain Management (SCM) problem we tackle in our research is how to coordinate the allocation, and monitoring of the activities of companies engaged in a supply chain process in order to have robust processes. A supply chain process, is defined by a set of activities that need to be performed in order to achieve a goal, such as producing and delivering a product.

The problem becomes even more complex due to dynamic nature of the events that occur in the environment. Communication networks can fail, trucks can break, schedules can slip, companies can have weak commitments towards the supply chain.

\subsection{Formalization}

We model the supply chain as a graph $S c=\langle A, C\rangle$. Where $A=\left\{a_{1}, a_{2}, \ldots, a_{n}\right\}$ is the set of agents representing partner companies, and $C \subseteq A \times A$ is the set of connections between agents.

The set of all possible service types offered by the companies participating in our supply chain is defined by $S=\left\{s_{0}, s_{1}, \ldots, s_{n}\right\}$, where $s_{i} \in \mathbb{N}$. Agents maintain a schedule indicating when they are busy. There is also a monetary price associated to the use of an agent. An agent $a_{i}$ is defined as:.

$$
a_{i}=\left(s_{i}, J_{i}, p_{i}\right) \text { where } \begin{cases}s_{i} \in S & \text { service type } \\ J_{i}=\left\{\left(t_{0}, e_{0}\right),\left(t_{1}, e_{1}\right), \ldots,\left(t_{n}, e_{n}\right)\right\} & \text { schedule } \\ p_{i} \in \mathbb{R}^{+} & \text {price }\end{cases}
$$

Where $s_{i}$ is the type of service offered by the agent. The agents maintain information about the times they will execute an operation and the expected duration time to perform such operation. This information is the agent schedule $J$, that is a set of tuples $\langle t, e\rangle$, where $t$ is the time to start executing an operation and $e$ is the expected duration to execute such operation. Finally, $p$ is the monetary price to use the agent service.

A process oriented supply chain constitutes of a number of activities that need to be executed in a specific order to create the desired product. For simplicity, we define a process $P=\left\{s_{i}, \ldots\right\}$, where $s_{i} \in S$, as a set of sequential service types that need to be invoked to complete the process.

When a distributor requests a new batch of products, it starts a process instance. A process instance can be seen as a path containing the expected time to start executing a service and the agent that will execute the service. A path is defined as

$$
\text { Path }=\left\{\left\langle t_{j}, a_{k}\right\rangle\right\} \text {, where } t_{j} \leq t_{j+1}, \forall t \in \mathbb{N} \text { and } a_{k}, \forall a_{k} \in A
$$

A given $P a t h$ is a valid path for the process $P$ if and only if for each pair $\langle t, a\rangle$, the agent $a$, associated to the service type $s_{i}$ from $P$, provides operations required by services of type $s_{i}$. This means that a path is valid if and only if the agents scheduled to participate in the path offer services of the same type required by the process. 
The expected completion time of a Path $=\left\{\left\langle t_{1}, a_{1}\right\rangle,\left\langle t_{2}, a_{2}\right\rangle, \ldots,\left\langle t_{n}, a_{n}\right\rangle\right\}$ is given by $t_{n}+\theta\left(a_{n}, t_{n}\right)$, where $\theta: A \times \mathbb{N} \rightarrow \mathbb{N}$ provides the time it takes to execute a task in a given agent $a_{n}$ at a given time $t_{n}$. Agents use the schedule information $J$ and the time information to define the function $\theta$.

The main problem we want to solve is to find a robust Path, illustrated in Equation 3 , while minimizing the costs associated to the selected path. The metric we use to define a robust path is the variation of total completion times.

$$
\min \sqrt{\frac{1}{n} \sum_{i=0}^{n}\left(\theta\left(a_{i}, t_{i}\right)-\overline{\theta(a, t)}\right)^{2}}
$$

\section{Decentralized Service Coordination}

In our approach, all supply chain companies participate, through their agents, in a service overlay network. This network is created according to trust relations, business contracts, between the companies. We assume that each agent maintains the information about its peers in the network. Hence there is no central entity used to store a map of the network.

Selecting services to participate in a supply chain process is a complex problem. We use Ant Colony Optimization (ACO) techniques in our coordination mechanism to tackle this complexity.

\subsection{Ant Colony Optimization}

ACO algorithms are inspired by food foraging behavior of real ant colonies. Ants are good at finding the shortest paths between their nest and sources of food. However they have little capacity to find the paths individually, they collaborate with each other to solve their food foraging problem. Ants drop pheromones in the environment leaving pheromone trails where they walk. Pheromones are chemical scents that stay in the air during a certain period. As more ants follow a certain path, they reinforce the pheromone trail on that path, leading to even more ants to follow that particular path [5].

In ACO a set of virtual ants, which are software agents, indirectly cooperate to find solutions to complex optimization problems. The ACO meta-heuristic defines a number of steps to create algorithms that mimic ant behavior. It can be applied to any problem that can be reduced to path-traversal problems.

The virtual ants have well defined behavior in order to contribute to finding solutions. Virtual ants drop information, called pheromones, along the paths that lead to good solutions. That way, other virtual ants, can smell the pheromone trail and follow it as well, converging to a path that represents a good solution to the problem at hand.

We use the pheromone concept to avoid direct communication between the agents, and also to indicate the quality of a certain path. We also use the concepts of virtual ants in our agents, called ExplorationAnts and IntentionAnts. We also use the probabilistic nature of the ants behavior to design our ExplorationAnts, what is explained next. 


\subsection{Decentralized Coordination and ACO}

Companies taking part in the service overlay network can have multiple participation modes: (i) service provider, which offer operations to other companies, (ii) service requester, which request operations from other companies.

A service provider is represented by a ResourceAgent. ResourceAgents are also responsible for storing the quality of service (QoS) information of the services they represent. Besides representing service providers, ResourceAgents maintain a reservation list indicating when their services will be used. This reservation list is frequently updated to avoid having stale information, i.e. in the case an agent does not intend to use the resource anymore. ResourceAgents also maintain information about their direct peers.

OrgAgents represent the service requesters. OrgAgents are responsible for selecting the services that will participate in a composition and are also responsible for maintaining the QoS of the composite service they represent. The main goal of OrgAgents is to create robust compositions, that is, enact supply chain processes with the best participants. OrgAgents delegate parts of their work to other agents, more specifically to ExplorationAnts and IntentionAnts, as TaskAgents in Delegate MAS.

ExplorationAnts and IntentionAnts are a special type of agent with constrained behavior. An OrgAgent sends out a number of ExplorationAnts to find out what are the best services available to accomplish the process at hand.

Once the ExplorationAnts find a valid path, they return this information to their OrgAgent. The OrgAgent collects the information brought by all ExplorationAnts and decides, based on its goals, to commit to one particular path. When an OrgAgent commits to a particular path, it sends out IntentionAnts that will reinforce the intention of the OrgAgent, making reservations, to use the services offered the ResourceAgents along the selected path.

ExplorationAnts main function is to search for ResourceAgents that can participate in a certain process. In order to do that, ExplorationAnts crawl the agent overlay network looking for ResourceAgents that: (i) offer the required type of service, (ii) can perform the service at the required time, and (iii) have a good QoS. The QoS is represented by the vector $q=\left(q_{1}, \ldots, q_{n}\right)$, where $q_{i} \in \mathbb{R}$ represents the quality $i$. The quality can be, for instance, the duration time to execute one operation, price, trustiness, etc. ExplorationAnts try to find a path containing agents capable of performing all the required operations needed by their OrgAgents. ExplorationAnts take QoS parameters into account, asking this information to ResourceAgents. They evaluate the QoS using an heuristic $\eta$, defined in Equation 4:

$$
\eta:\left(q_{1}, \ldots, q_{n}\right) \rightarrow \mathbb{R}, \text { such that } \eta\left(\left(q_{1}, \ldots, q_{n}\right)\right)=\frac{1}{\sum_{i}^{n} q_{i}^{*}}, q_{i}^{*} \text { is the normalized } q_{i}
$$

where $\eta$ is evaluated every time an ExplorationAnt checks for the quality of a service represented by a given ResourceAgent. An ExplorationAnt decides on which path to follow according to the probability given by Equation 5: 


$$
P_{i j}(t)=\frac{\left[\tau_{i j}(t)\right]^{\alpha}\left[\eta_{i j}(t)\right]^{\beta}}{\sum_{l \in N_{i}}\left[\tau_{i l}(t)\right]^{\alpha}\left[\eta_{i l}(t)\right]^{\beta}}
$$

where $\tau$ is the pheromone level, $\alpha$ indicates how much the ExplorationAnt values the pheromone information, $\eta$ is the heuristic that takes the QoS into account, and $\beta$ indicates the weight to the QoS information the ExplorationAnt gives.

ExplorationAnts select a path to follow according to the probabilities defined in Equation 5. There is always room for exploratory behavior, unless the parameter $\alpha$ is set to high. If $\alpha$ is set to high, ExplorationAnts will only follow the paths with the highest pheromone concentration. ExplorationAnts jump from ResourceAgent to ResourceAgent, either until their time to live expires or they have found a valid path, that is, they have found all the required ResourceAgents needed for the composition.

When an OrgAgent decides to follow a particular path, it sends out IntentionAnts. IntentionAnts make reservations to use the services of the selected ResourceAgents. Another function of IntentionAnts is to drop pheromones along the chosen path in the agent overlay network. The pheromone information is used by ExplorationAnts, when they are looking for services.

If a pheromone trail becomes to strong, ExplorationAnts will have a higher probability to follow this trail instead of exploring new solutions. We use a mechanism called Pheromone Evaporation that decreases the pheromone level associated to a certain path as time pass by.

\section{Evaluation}

This section shows the experimental results that we had simulating our coordination mechanism in different scenario settings. As explained in Section 3 there are two types of agents operating in the overlay network, ResourceAgents and OrgAgents, and two types of ants, ExplorationAnts and IntentionAnts. All ResourceAgents are bootstrapped with the same parameter configurations. OrgAgents are bootstrapped with the same commitment level.

In our simulation's scenario there are 20 agents of type Manufacture1 (mean response $110 \mathrm{~ms} \pm 11 \mathrm{~ms}$ ), 100 agents of type TransportRegion1 (mean response time $300 \mathrm{~ms} \pm 30 \mathrm{~ms}$ ), 20 agents of type Manufacture 2 (mean response time $80 \mathrm{~ms} \pm 8 \mathrm{~ms}$ ), 100 agents of type TransportRegion 2 (mean response time $300 \mathrm{~ms} \pm 30 \mathrm{~ms}$ ), and 8 OrgAgents. Due to the probabilistic nature of our coordination mechanism we performed 30 simulations per experiment to obtain statistically significant results with a $95 \%$ confidence interval.

\subsection{Static Environment}

In this experiment, services never fail and have an execution time given by the Poisson probability distribution as describe above. OrgAgents are configured with a commitment level of 0.0 . The compositions created by our coordination mechanism had a average response time of $790 \mathrm{~ms} \pm 80 \mathrm{~ms}$, basically resembling the nature of the component services. With this values at hand we decided to investigate how different OrgAgent's 
commitment strategies could influence the created compositions. What is explained in the OrgAgent sensitivity analysis.

Sensitivity Analysis of OrgAgent's Commitment Levels influence the quality of created compositions. A commitment level of 1.0 indicates that the OrgAgent performs the component service selection only once and never changes this selection, it is called a blinded committed agent. A blind committed OrgAgent is unable cope with unexpected changes in the environment, since it never changes its component services.

A cautious agent has a commitment level of 0.0. It revises its commitments every cycle, what can incur in instability in the system, since the agent can keep alternating between component services and never engage with any one.

Figure 1a indicates the average response time of the created compositions by instantiating OrgAgents with different commitment levels, in a static environment.

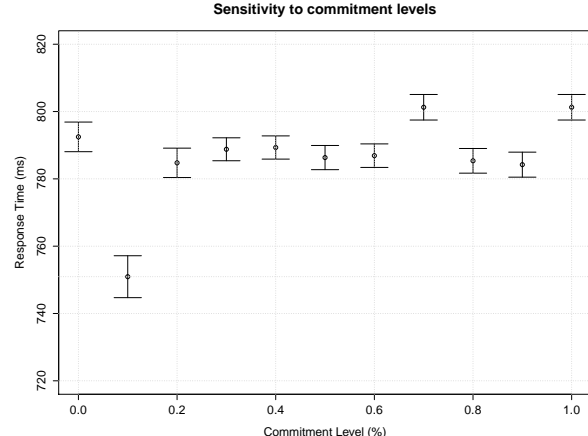

(a) Commitment level between 0.0 and 1.0

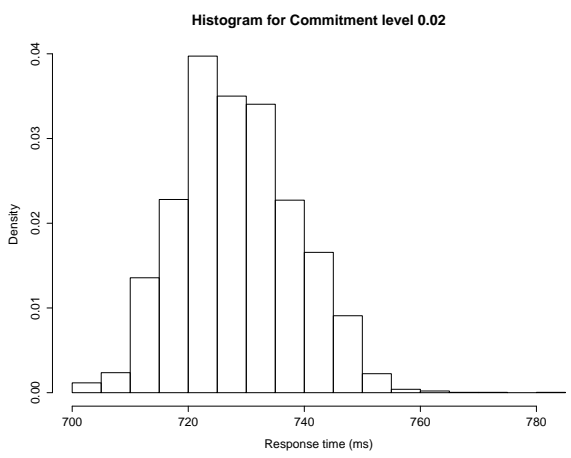

(c) Static environment

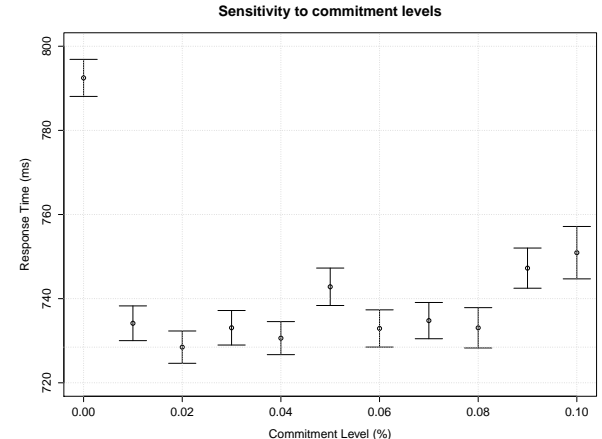

(b) Commitment level between 0.0 and 0.1

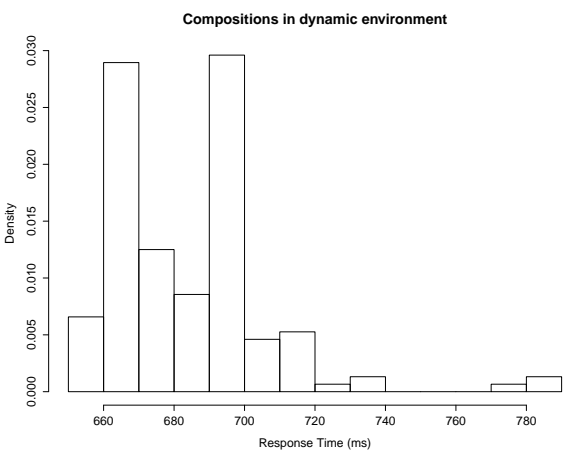

(d) Dynamic environment

Fig. 1: Sensitivity analysis of OrgAgent's commitment levels and simulation results. 
Given the results obtained we found that a commitment level of 0.02 gives the best compositions for this scenario, depicted in Figure 1b. A commitment level of 0.02 indicates that an agent will change its path if the increment in the solution is greater than $2 \%$.

The quality of the compositions created by the OrgAgents with a commitment level of 0.02 increased by a factor of more than $10 \%$, what is depicted in Figure 1c. It is interesting to note that the standard variations were also minimized when the OrgAgent had their commitment levels changed to 0.02 .

\subsection{Dynamic environment}

The reality has many sources of dynamic events, such as network failures, failures to keep commitments, even failures to properly predict schedules. To approximate our simulations to the reality we performed simulations with our coordination mechanism in a dynamic environment. We randomly, using the Uniform probability distribution, fail services that are participating in the agent overlay network at a rate of 1 service failure per 10 iterations. The OrgAgents were configured with commitment level of 0.02. The results are depicted in Figure 1d.

It can be seen from Figure 1d that the coordination mechanism can still create responsive service compositions, in the presence of component service failures. However we should note that the objective to minimize the standard deviation is hard to achieve, since a number of services are failing and the remaining services can have greater differences in their QoS values, in this case response time.

\section{Related Work}

There is plenty of research about selecting component services to participate in service compositions. These works focus on creating algorithms capable of selecting the best available component services, in terms of QoS. Our work shares the idea that it is possible to improve properties from composite services by selection and biding to component services at runtime. However, in our research we study the problem of not only selecting the best available services, but also selecting component services that will lead to robust compositions.

The work presented in [6] discusses and evaluate different techniques for component service selection. The simulation results show that the most efficient approach is the proxy-based, followed by the collaborative approach. In the proxy-based approach, all the service invocations go through a proxy that can then, load balance and select the best available services. In the collaborative approach different composite services collaborate, sharing QoS information about component services, to allow a better component service selection.

Our solution shares characteristics with both the proxy-based and collaborative approaches. We focus, however, on the creation of robust compositions instead of focusing only on minimizing a certain QoS metric, such as response time or price.

The work on [7] explicitly focus on creating robust service compositions. The work uses decision theory for dealing with the uncertainty associated with component service providers. It proposes a mechanism for component service selection that explicitly 
takes the reliability of the created composition into account. The service selection algorithm takes the most critical tasks into account and use service redundancy for these tasks. The algorithm also uses planning techniques to create contingency plans, in the case of component services failures. Another interesting characteristic of the algorithms presented in [7] is the use of service reservation for parts of the composite service.

Our work also takes the robustness of the composition into account when selecting component services. However our approaches differ in how to create robust compositions. Our approach relies on the aggregated information available in the agent overlay network and in advanced reservations of services that will participate in the composition.

\section{Conclusions and Future Work}

The main contribution of this work is to present a decentralized coordination mechanism capable of creating robust service compositions. We have strong indications that our mechanism can be used to create collaborative systems that need to support companies interacting in a supply chain.

We should note that supply chains are quite irregular in reality what was not explored in our current work, but should, definitely, be explored in our future works.

A future research is to evaluate how adding autonomic behaviour to our OrgAgents, for instance, changing the commitment levels at runtime, can influence the quality of the created compositions. Finally we intend to evaluate our coordination mechanism in a real network environment.

\section{Acknowledgments}

This research is partially funded by the Interuniversity Attraction Poles Programme Belgian State, Belgian Science Policy, and by the Research Fund K.U.Leuven.

\section{References}

1. E. Sandberg and M. Abrahamsson, "The role of top management in supply chain management practices," International Journal of Retail \& Distribution Management, vol. 38, no. 1, pp. 57 69, 2010.

2. R. Glenn Richey, M. Tokman, and V. Dalela, "Examining collaborative supply chain service technologies: a study of intensity, relationships, and resources," Journal of the Academy of Marketing Science, vol. 38, no. 1, pp. 71-89, 2010.

3. H. Ludwig, T. Nakata, O. Wäldrich, P. Wieder, and W. Ziegler, "Reliable orchestration of resources using ws-agreement," in High Performance Computing and Communications, ser. Lecture Notes in Computer Science, M. Gerndt and D. Kranzlmüller, Eds. Springer Berlin / Heidelberg, 2006, vol. 4208, pp. 753-762.

4. T. Holvoet, D. Weyns, and P. Valckenaers, "Patterns of delegate mas," Self-Adaptive and SelfOrganizing Systems, International Conference on, vol. 0, pp. 1-9, 2009.

5. M. Dorigo, G. D. Caro, and L. M. Gambardella, "Ant algorithms for discrete optimization," Artificial Life, vol. 5, pp. 137-172, 1999. 
6. C. Ghezzi, A. Motta, V. Panzica La Manna, and G. Tamburrelli, "Qos driven dynamic binding in-the-many," in Research into Practice - Reality and Gaps, ser. Lecture Notes in Computer Science, G. Heineman, J. Kofron, and F. Plasil, Eds. Springer Berlin / Heidelberg, 2010, vol. 6093, pp. 68-83. [Online]. Available: http://dx.doi.org/10.1007/978-3-642-13821-8_7

7. S. Stein, T. Payne, and N. Jennings, "Robust execution of service workflows using redundancy and advance reservations," Services Computing, IEEE Transactions on, vol. 4, no. 2, pp. 125 -139 , feb. 2011. 